\title{
Carbon Molecular Sieve Membranes from Polyetherimide: Effect of Pyrolysis Temperature on $\mathrm{O}_{2} / \mathrm{N}_{2}$ Selectivity
}

\author{
M. A. Ahmad ${ }^{1 *}$, N. K. A. Rashid ${ }^{2} \&$ B. H. Hameed ${ }^{3}$ \\ ${ }^{1,2 \& 3}$ School of Chemical Engineering, Engineering Campus, Universiti Sains Malaysia, 14300 Nibong Tebal, \\ Penang, Malaysia
}

\begin{abstract}
Carbon molecular sieve (CMS) membranes with excellent separation performance and stability appear to be promising candidate for gas separation. In this work, CMS membranes were formed by a thin carbon layer obtained by pyrolysis of a coated polyetherimide solution onto porous disk support. The pyrolysis temperatures were varied under inert condition. Results showed that the pyrolysis temperature played an important role in determining the gas permeability of CMS membranes. The CMS membrane prepared at pyrolysis temperature of $700^{\circ} \mathrm{C}$ shows high surface area and narrow PSD with well developed microporous carbon structures. The development of large pore occurs at higher pyrolysis temperature. The $\mathrm{O}_{2} / \mathrm{N}_{2}$ permselectivities of $2.86,2.61$ and 2.22, respectively were attained by CMS membranes prepared at pyrolyzed temperature of 700,800 and $900^{\circ} \mathrm{C}$.
\end{abstract}

Keywords: Carbon molecular sieve membranes, gas permeation, pyrolysis, polytherimide

\subsection{INTRODUCTION}

Carbon molecular sieve (CMS) membranes have received much attention for gas separation due to its superior gas permeation performance as well as high thermal and chemical stability [1]. Furthermore, CMS membranes are amorphous in nature. In addition, CMS membranes are tougher and more flexible [2]. For gas separation process, the membranes needs to exhibit molecular sieving capabilities with pore size near the dimension of gas molecules to be separated [3]. CMS membranes contain a thin carbon layer having pores with a size smaller than $1 \mathrm{~nm}$, which allow the gases separation such as oxygen $\left(\mathrm{O}_{2}\right)$ and nitrogen $\left(\mathrm{N}_{2}\right)$ from air [4]. Pure $\mathrm{O}_{2}$ is widely used for medical purposes while pure $\mathrm{N}_{2}$ is used for blanketing perishable fruits and also for shipment of flammable liquids. Suda and Haraya [5] pyrolyzed Kapton polyimide at $800^{\circ} \mathrm{C}$ and obtained CMS membrane

\footnotetext{
*Corresponding author: M. A. Ahmad (email: chazmier@ eng.usm.my)
}

with $\mathrm{O}_{2} / \mathrm{N}_{2}$ selectivity of 12 . Ghosal and Koros [6] found that the CMS membrane prepared from pyrolysizing hexafluoroisopropylidene (6-FDA)based copolyimide at $800^{\circ} \mathrm{C}$ exhibit high $\mathrm{O}_{2} / \mathrm{N}_{2}$ selectivity of 12 . In this work, polyetherimide was used for CMS membranes preparation for $\mathrm{O}_{2}$ and $\mathrm{N}_{2}$ gas permeation study. Polyetherimide has excellent physical properties and easily tunable the chemical composition by utilizing different molecular structures composed by dianhydride and diamine monomers [1].

\subsection{MATERIALS AND METHODS}

\subsection{Materials}

The phenolic resin and polyetherimide were purchased from Sigma Aldrich (M) Sdn. Bhd. The cellulose acetate and $\mathrm{N}$-methylpyrrolidone (NMP) were purchased from Acros Organics Ltd. The powdered fine carbon black was supplied by CarboTech $\mathrm{GmbH}$. Purified $\mathrm{O}_{2}$ (99.95\% purity) 
and $\mathrm{N}_{2}$ gases (99.99\% purity) were supplied by Air Product (M) Sdn Bhd.

\subsection{Membrane Preparation}

The porous membrane support was made by blending phenolic resin $(60 \%)$ and carbon black (30\%) together with cellulose acetate $(10 \%)$ as binder. This blend was pressed at 1200 bar in a static press resulting in disk-shaped support of $20 \mathrm{~mm}$ in diameter and $2.5 \mathrm{~mm}$ in thickness. Meanwhile the polymer solution which consists of $9 \mathrm{wt} \%$ polyetherimide and $91 \mathrm{wt} \%$ NMP was stirred for $4 \mathrm{~h}$ to prepare a clear yellowish solution before coated onto the support by dip coating method and pyrolyzed to form molecular sieve layer. The sample was pyrolyzed under purified $\mathrm{N}_{2}$ flow $(99.99 \%)$ to a final temperature of 700 , 800 and $900^{\circ} \mathrm{C}$ (denoted as CMS700, CMS800 and CMS900, respectively) at a rate of $2^{\circ} \mathrm{C} / \mathrm{min}$ for $3 \mathrm{~h}$.

\subsection{Permeation Measurements}

Gas permeation measurement was performed using the variable volume-constant pressure rig set up which was developed for laboratory scale. Each sample was treated prior to an elevated temperature at $100^{\circ} \mathrm{C}$ for $10 \mathrm{~min}$ to ensure that all water vapor trapped on the carbon surface was completely vaporized. The system was maintained 2.5 bar at feed section and vacuum at the permeate section. The variable volume-constant pressure method was applied for gas permeation test. Calibrated soap film flow meter was used to obtain the gas permeance penetrate traverse the membrane. The permeance was estimated using equation below [6]:

$$
P=\frac{Q}{\Delta P \times A}
$$

where $Q$ is the gas flow rate $(\mathrm{mol} / \mathrm{s}), \Delta P$ is the difference in the pressure of gases across the membrane ( $\mathrm{Pa}$ ) and $A$ is the membrane's effective surface area of membrane $\left(\mathrm{m}^{2}\right)$.

The selectivity of the membrane to specific gas is subject to the ability of the molecule to diffuse through the membrane. The permselectivity or ideal selectivity factor $\alpha$, for $\mathrm{O}_{2}$ and $\mathrm{N}_{2}$ gases is defined as:

$$
\alpha_{O_{2} / N_{2}}=\left(\frac{P_{O_{2}}}{P_{N_{2}}}\right)
$$

\subsection{Membrane Characterization}

The surface area, total pore volume micropore volume and pore size distribution of the sample were characterized by $\mathrm{N}_{2}$ adsorption at $77 \mathrm{~K}$ using Micromeritics ASAP 2020 volumetric adsorption apparatus. The surface area was determined using Brunauer-Emmett-Teller (BET) equation. DubininRadushkevich (DR) equation was used to calculate the micropore volume over a range of relative pressure of $1.1 \times 10^{-5}$ to 0.02 . The total pore volume $\left(V_{t}\right)$ was obtained at a relative pressure of 0.99. Pore size distribution (PSD) was obtained from Horvath-Kawazoe (HK) analysis. The functional group of the sample was estimated by Fourier transform infrared (FTIR) spectroscopy (FTIR-2000, Perkin Elmer). The surface morphology of the samples was examined using a scanning electron microscope (FESEM Leo Supra 40VP).

\subsection{RESULTS AND DISCUSSION}

\subsection{CMS Membranes Characterization}

Table 1 shows the properties of CMS membranes. As the pyrolysis temperature increases, the surface area and pore volume decreases. Sample CMS700 shows narrower PSD at approximately $0.41 \mathrm{~nm}$ compared to CMS800 and CMS900 as shown in Figure 1. As the pyrolysis temperature increases, the curve peak shifts to the right giving wider PSD with pore size range of $0.45-0.49 \mathrm{~nm}$. In addition, the differential pore volume $(\mathrm{d} V / \mathrm{dw})$ decreases

Table 1 CMS membranes properties

\begin{tabular}{lcccc}
\hline Sample & $\begin{array}{c}\mathrm{S}_{\mathrm{BET}} \\
\left(\mathrm{m}^{2} / \mathrm{g}\right)\end{array}$ & $\begin{array}{c}\mathrm{V}_{\mathrm{t}} \\
\left(\mathrm{cm}^{3} / \mathrm{g}\right)\end{array}$ & $\begin{array}{c}\mathrm{V}_{\text {mic }} \\
\left(\mathrm{cm}^{3} / \mathrm{g}\right)\end{array}$ & $\begin{array}{c}\mathrm{D}_{\text {mean }} \\
(\mathrm{nm})\end{array}$ \\
\hline CMS700 & 427.3 & 0.377 & 0.351 & 0.41 \\
CMS800 & 381.1 & 0.391 & 0.330 & 0.45 \\
CMS900 & 334.8 & 0.428 & 0.303 & 0.49 \\
\hline
\end{tabular}




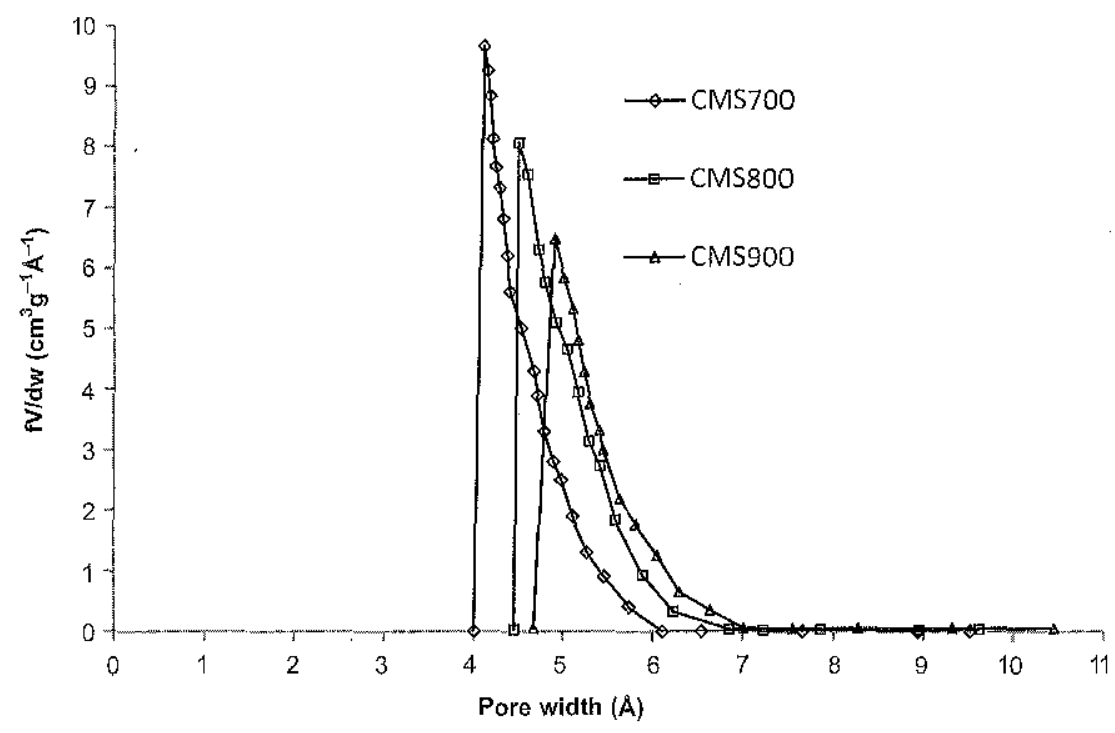

Figure 1 Pore size distribution of CMS membranes

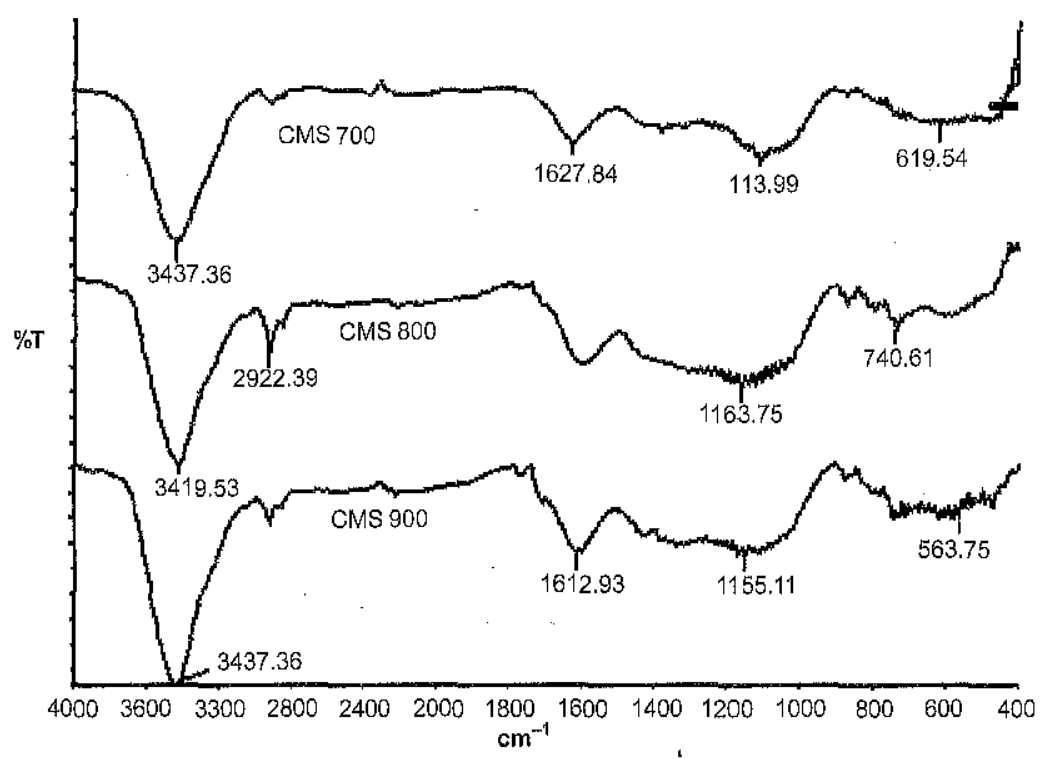

Figure 2 FT-IR spectra of CMS membranes

from 9.65 to $6.48 \mathrm{ml} / \mathrm{g} \mathrm{nm}$. At higher pyrolysis temperature, the development of the undesirable large pore begins by the burn out of walls between adjacent micropores [7].

The FTIR spectra shown in Figure 2 indicates that the peak intensities for the aliphatic or aromatic O-H $\left(3200-3500 \mathrm{~cm}^{-1}\right), \mathrm{C}-\mathrm{H}(2800-$ $\left.3200 \mathrm{~cm}^{-1}\right), \mathrm{C}=\mathrm{O}\left(\sim 1700 \mathrm{~cm}^{-1}\right), \mathrm{C}-\mathrm{N}(1100-1200$ $\left.\mathrm{cm}^{-1}\right)$ and benzene ring $\left(<750 \mathrm{~cm}^{-1}\right)$ exist in CMS membranes. The results suggest that although the PSD of the CMS membranes pyrolyzed at $700-$ $900^{\circ} \mathrm{C}$ were different but the chemical structures were almost unchanged.

Figure 3 shows the morphology of the sample CMS700 from top view and cross section angle. Two different parts can be distinguished, which were microporous carbon film and porous carbon support. The carbon film constitutes a dense 


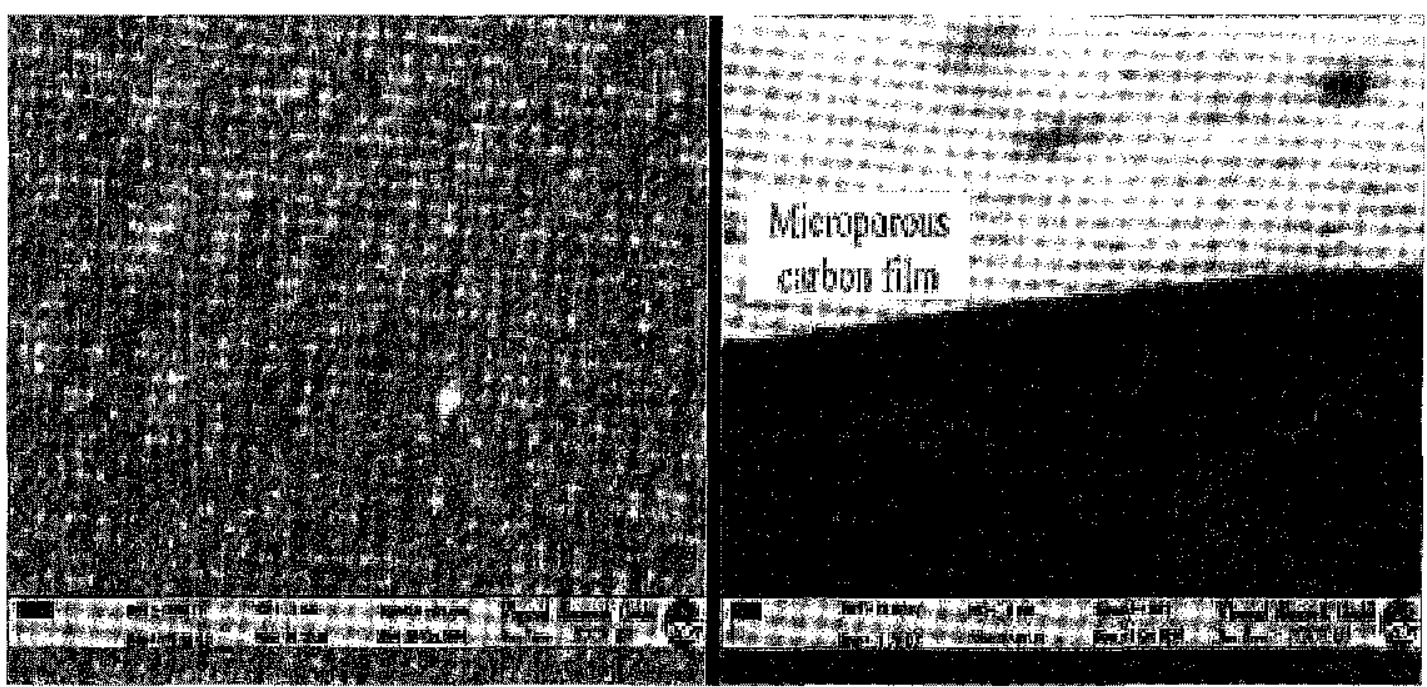

Figure 3 SEM images of CMS700; (a) top surface (117.8k $x$ ) and (b) cross sectional view (1.75k $\times$ )

layer with a thickness around $5 \mu \mathrm{m}$ and uniform microporous matrix form by the pyrolysis of polyetherimide. The top layer was very smooth and almost defect-free. The interface between the dense skin layer and the porous matrix was very sharp. A good adherence between the homogeneous porous matrix and the carbon support can be observed.

\subsection{Gas Permeation Performance}

The gas permeation result of the CMS membranes pyrolyzed at 700,800 and $900^{\circ} \mathrm{C}$ are shown in Figure 4. From the figure, the $\mathrm{O}_{2}$ has better gas

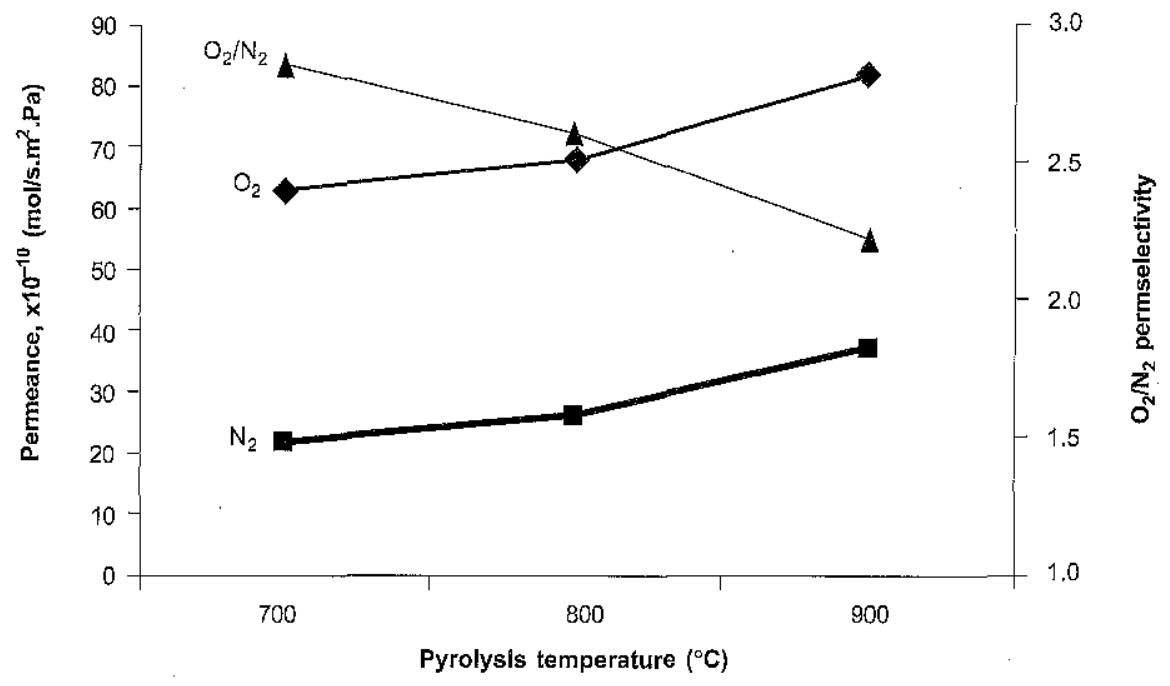

Figure 4 Gas permeance and $\mathrm{O}_{2} / \mathrm{N}_{2}$ permselectivity of CMS membranes 
permeability than $\mathrm{N}_{2}$. This was due to the comparatively higher rate of diffusion of the $\mathrm{O}_{2}$ molecule (molecular diameter $=0.346 \mathrm{~nm}$ ) into the CMS micropores than $\mathrm{N}_{2}$ molecule $(0.364 \mathrm{~nm})$ [4]. The $\mathrm{O}_{2}$ and $\mathrm{N}_{2}$ gas permeance increased with increased in pyrolysis temperature due to the formation of microporous within the carbon structure. The increase in pyrolysis temperature will also lead to a carbon membrane with higher compactness, a more turbostratic structure, higher crystallinity and density, and smaller average interplanar spacing between the graphite layers of the carbon [8]. However, as the pyrolysis temperature increased, the permselectivity decreased due to enlargement pores of the CMS membrane. Both gases can penetrate easily which result in decreased in permselectivity. The permselectivity for $\mathrm{O}_{2} / \mathrm{N}_{2}$ were $2.86,2.62$ and 2.22 for pyrolysis temperature of 700,800 and $900^{\circ} \mathrm{C}$, respectively.

\subsection{CONCLUSIONS}

At pyrolysis of $700^{\circ} \mathrm{C}$, the CMS membrane prepared shown high surface area and narrow PSD with well developed microporous carbon structures. At higher pyrolysis temperature, the surface area and pore volume decreased due to the development of large pore, which resulted in decreased in $\mathrm{O}_{2} / \mathrm{N}_{2}$ permselectivity. Sample CMS700 gave the highest $\mathrm{O}_{2} / \mathrm{N}_{2}$ permselectivity at $6.3 \times 10^{-9} \mathrm{~mol} / \mathrm{s} . \mathrm{m}^{2} . \mathrm{Pa}$ and 2.86 , respectively.

\section{ACKNOWLEDGEMENT}

The authors acknowledge the research grant provided by MOSTI (PJKIMIA/6013373) and Universiti Sains Malaysia under the Fundamental
Research Grant Scheme (PJKIMIA/6070015) that resulted in this article.

\section{REFERENCES}

[1] Youn, K. K., B. P. Ho, and M. L. Young. 2005. Preparation and Characterization of Carbon Molecular Sieve Membranes Derived from BTDA-ODA Polyimide and Their Gas Separation Properties. J. Membr. Sci. 225: 265-273.

[2] Wang, L. J., and F. C. N. Hong. 2005. Effects of Surface Treatments and Annealing on Carbon-Based Molecular Sieve Membranes for Gas Separation. Applied Surface Science. 240: 161-174.

[3] M. A. Ahmad. 2009. Preparation of Carbon Molecular Sieves from Palm Shell: Effect of Benzene Deposition Conditions. Adsorption. 15: 489-495.

[4] M. A. Ahmad, W. M. A. Wan Daud, and M. K. Aroua. 2008. Adsorption Kinetics of Various Gases in Carbon Molecular Sieves (CMS) Produced from Palm Shell. Colloids and Surfaces A: Physicochemical and Engineering Aspects. 312: 131-135.

[5] Suda, H., and K. Haraya. 1995. Molecular Sieving Effect of Carbonized Kapton Polyimide Membrane, J. Chem Soc Chem Commun. 15: 1179-1180.

[6] Ghosal, A. S., and W. J. Koros. 2000. Air Separation Properties of Flat Sheet Homogenous Pyrolytic Carbon Membranes. J. Membr. Sci. 174: 177-188.

[7] M. A. Ahmad, W. M. A. Wan Daud, and M. K. Aroua. 2007. Synthesis of Carbon Molecular Sieves from Palm Shell by Carbon Vapor Deposition. J. Porous Mater. 14: 393-399.

[8] Saufi, S.M., and A.F. Ismail. 2004. Fabrication of Carbon Membranes for Gas Separation - A Review. Carbon. 42: 241 259. 